Journal of Computer Science 8 (5): 716-720, 2012

ISSN 1549-3636

(C) 2012 Science Publications

\title{
Segmentation of Ureteric and Bladder Calculi in Ultrasound Images
}

\author{
Sridhar, S. \\ Department of Information Science and Technology, \\ Faculty of Information and Communication Engineering, \\ Anna University, Chennai, 600 025, India
}

\begin{abstract}
Problem statement: The framework for segmenting calculi in ureter and bladder using ultrasound images is proposed in this study. Calculi are due to abnormal collection of certain chemicals like oxalate, phosphate and uric acid. These calculi can be present in kidney, urethra or in urinary bladder. The extraction of calculi in medical images is a difficult task as no standard algorithms are available. This poses a serious a problem for successful diagnosis of calculi disease. The proposed technique is specific for the extraction of calculi in ureter and bladder. This constitutes the first stage in the successful treatment of calculi disease. Approach: An algorithm is proposed to detect calculus based on the shadow it casts in ultrasound image. Calculi are present in ultrasound images as bright spots. But noise in the image also can be bright spots. So it is easy to misinterpret the presence of noise as calculi. The proposed framework thus has two phases. In phase one, five standard algorithms are modified and are used to segment the bright spots present in the ultrasound images using the intensity profile. Calculi are having intensity in the range of 10-40. So all the potential calculi as well as the noise that appear as bright spots are segmented in phase one. In phase two,a validation procedure is used to validate the presence of calculi using its acoustic shadow property in the ultrasound images. Results: Ultrasound images of twenty-seven ureteric and bladder calculi patients are used for testing the framework. The detected calculi by the proposed framework are validated against a group of experts. The Performance of the proposed method is thoroughly investigated and the accuracy of the framework is determined. The framework incorporating automated seed selected region growing algorithm is able to detect the calculi with the efficiency of 78.57\%. Conclusion: The extracted calculi can further be analyzed for taking decision about the treatment procedures. The proposed system is helpful for taking decision about the treatment procedures. The proposed system is helpful for clinicians as a decision support tool. This system can also be useful as educational aid for assisting or decision making in the treatment of calculus disease.
\end{abstract}

Key words: Kidney stones, ureteric calculi, bladder calculi, automatic segmentation, ultrasound images, decision support systems

\section{INTRODUCTION}

Calculi are due to abnormal collection of certain chemicals like oxalate, phosphate and uric acid. Ultrasound is the usual image modality for detecting the presence of calculi in ultrasound images. The most common type of calculi contains calcium in combination with either oxalate or phosphate. The various types of calculi are, 1. Oxalate (These are single, hard, rough and cast a good shadow), 2. Phosphate (These are smooth, dry white and bigger in size), 3. Uric acid and Urate (These are numerous in number, white and translucent) (Malvinder, 2004; Duncan and Ayache, 2000).
Segmentation of calculi in ultrasound images is prerequisite for taking decision regarding treatment. Segmentation technique basically divides the spatial, on which the image is defined, into meaningful parts or regions. This meaningful region may be a complete object or part of it. The available segmentation algorithms are general techniques and fail to detect calculi in ultrasound images. The main reason is the presence of high noise in ultrasound images, which manifests itself as white patches. It is therefore possible that the noise may be incorrectly diagnosed as calculi. A validation is required for proper identification of calculi and gallstones. As a result, there exists no general segmentation algorithm in hand that is suitable 
for segmentation. Standard segmentation algorithms fail as they are ad hoc in nature.

An algorithm is proposed by the authors (Sridhar $e t$ al., 2002) for the detection of renal calculi. This proposed segmentation framework validation criterion (Sridhar et al., 2002) is based on the presence of acoustic shadow caused by the calculi in ultrasound images. The strength of the shadow depends on the physical characteristics of renal calculi. This framework is extended to include ureteric and bladder in ultrasound images.

The first step in image processing is the acquisition of the ultrasound image. In Ultrasound method, pulses of ultrasound are transmitted into the body by placing the ultrasound transducer in close contact with the skin using a jelly paste or water to eliminate the air so as to provide proper impedance matching at the skin and to increase the transmission of the ultrasound into the body. The reflected echoes are displayed on the screen as an image.

\section{MATERIALS AND METHODS}

Related works: The problem of segmenting the bright spots is addressed in various literatures. The most popular algorithms are thresholding technique (Sridhar, 2011), which is used to segment regions of interest based on histograms. Edge-based techniques are also mentioned in the literature (Marques, 2011) for image segmentation. Region growing techniques are also useful for image segmentation. This is improved by Pyramidal seeded region growing algorithm (Tomori et al., 1999) uses multiple seeds that grow simultaneously for segmenting the region of interest. Morphology based segmentation (Tamilselvi and Thangaraj 2012) is also useful in extracting the calculi present in the ultrasound images. An attempt is also made to use active contours or snakes to find object boundaries (Umbaugh, 2010). These are computer generated curves that mode within images to explore the boundaries.

Proposed method: The ultrasound image of the patient is acquired. Ultrasound images may contain noise that makes the segmentation process less accurate. So the image is processed so that the resultant image is more suitable than the original for the application of the proposed algorithm. This process involves background removal and filtering. The background removal is done using background subtraction algorithm (Sridhar, 2011). The noise is then removed using median filter (Umbaugh, 2010). Since the density of the calculi much higher, calculi are always brighter than the neighboring pixels.
A framework is proposed (Sridhar et al., 2002) which combine an effective algorithm to detect the brighter calculi and a validation process for detecting the calculi. So the algorithm has two phases.

In the first phase, all bright spots are identified as potential calculi using the algorithms, automatic segmentation (Marques, 2011), active contour models (Umbaugh, 2010), Pyramidal seeded region growing algorithm (Tomori et al., 1999), Morphology based segmentation (Tamilselvi and Thangaraj, 2012), Merge and split (Marques, 2011) and region growing algorithm (Sridhar, 2011). The object contour is then encoded, smoothened and connected. A seed fill algorithm is used to fill in the border contour. But all the bright spots are not calculi. An automatic algorithm is proposed based on the region-growing framework. The algorithm chooses the initial seed automatically. It has been observed that all calculi are bright spots. Based on the histogram, it is observed that they have gray values in the range (230-255). Using this fact, an automatic seed selected region-growing algorithm is proposed.

The pseudo code of the algorithm is given below Algorithm Automatic seed selected region-growing algorithm:

Step $1=$ Read the Image

Step 2 = Scan the image line by line from the image top left to the image bottom right (a) Search for a pixel having a higher intensity value, called the seed (b) Find the neighbor pixel using connectivity. If the neighbor has the value within the tolerance, add the pixel and grow the region (c) Repeat step $2 \mathrm{~b}$ till a complete region of similar intensity of connected region is obtained.

This algorithm is useful for locating the bright spots. The algorithm however, is not effective in detecting calculi. Hence a framework is suggested for the detection of calculi in ureter and bladder. The constructed framework consists of two phases. In the first phase, all the bright spots are identified as potential calculi. But all the bright spots are not calculi. So in phase two, the bright spot is validated based on the presence of its shadow.

Proposed segmentation framework for renal calculi and gallstones detection: The proposed framework has two phases.

First phase: In the first phase, a proposed algorithm along with five already existing methods is used to segment the bright spots, which include calculi and noise pixels. The proposed automatic algorithm 
(Sridhar et al., 2002) is used along with the existing algorithms like active contour models, Pyramidal Seeded Region growing algorithm; Morphology based watershed segmentation, Merge and split and region growing algorithms for detecting bright spots. The object contour is encoded using the Freeman chain code (Umbaugh, 2010). After the border contour has been vectorized, the contour is smoothened by sub sampling the vector data and using a B-spline to connect the points (Umbaugh, 2010). A seed fill algorithm is then used to fill in the border contour.

Second phase: Once a prospective calculi region is identified, the validity of the calculi is tested. The property of the ultrasound imaging process is that the shadow is always below the calculi in the image. So, it is necessary to check the shadow below the prospective calculi. The potential region of the shadow is narrowed down to a smaller portion of a polygon which is constructed by taking the extreme points of the major diagonal axis of the potential calculi and extending it downwards till the last line of the image.

Now the region of searching for shadows is limited within the polygon. A mask of $3 \times 3$ grid of unit weight is used to detect the shadow.

The shadow regions of all the images are studied and are found to have gray value in the range of 10-40. These are characterized by the variables, shadow_min and shadow_max. The above mask is convolved with the image and if the convolved output is in the range $10-40$, then the point is assumed to be a shadow point. If the shadow points are connected in the neighborhood, they form a uniform shadow.

The complete algorithm for the proposed framework is as below:

Algorithm: (Detection of Calculi):

Step $1=$ Read the ultrasound Image

Step $2=$ [Phase one] Detect the potential calculi as reported in the first phase.

Step $3=$ [Phase two] (a) Position the region and go below the region of interest and form a polygon determined by the extreme points in the major diagonal axis. (b) Find the convolved value by applying a 3 X 3 mask of unit weight within the polygon. If the value is between shadow_min and Shadow_max, consider it as a shadow point (c) If the shadow points are connected in the neighborhood, report that calculi are present:

Step $4=$ Exit.
So in Phase two, the bright spot is validated based on the presence of shadow using the proposed shadow mask.

\section{RESULTS}

The performance of the semiautomatic and automatic methods presented in the framework is evaluated and compared using the following criteria.

- $\quad$ Success rate of the framework in detecting calculi by using sensitivity and specificity analysis

- Accuracy of the border detection of the calculi

- Time considerations

For evaluating the framework, twenty-seven Ureteric and bladder patients' images are used. The case histories of the patients are shown in Table 1. A panel of three experts has been involved in validating the images manually. The three experts read the images independently and arrived at the consensus. The agreement variation patterns are listed in Table 2. Sensitivity and specificity analysis are carried out and the results are shown in Table 3.

Accuracy of the border estimation: The images features like size and area of the calculi is crucial for predicting the treatment. Hence, the accuracy of the object boundary detection is very important.

The success of the border segmentation relative to the true border determined by the expert clinician is denoted by a metric called border error metric (Umabaugh S., 2011). It is defined as:

$$
\frac{\operatorname{Area}\left(I_{a}(r, c) \otimes I_{b}(r, c)\right)}{\operatorname{Area}\left(I_{a}(r, c)\right)}
$$

where, $\otimes$ denotes the Exclusive-Or operator. $\mathrm{I}_{\mathrm{a}}(\mathrm{r}, \mathrm{c})$ and $\mathrm{I}_{\mathrm{b}}(\mathrm{r}, \mathrm{c})$ are both binary images representing the actual and segmented border. The area denotes a two dimensional binary area. This metric gives a zero if there is a perfect match between the two borders. Its value is one if there is a total mismatch. Otherwise, the values will be in the range $0-1$. Table 4 shows the border error metric values obtained using various segmentation algorithms in the framework

Table 1: Case history based on manual interpretation of ureteric and bladder patients

\begin{tabular}{lc}
\hline Number of patients & As per experts 1,2 and 3 \\
\hline Patients with no calculi & 6 \\
Patients with a single calculus & 20 \\
Patients with multiple calculi & 1 with two calculi \\
Total calculi detected & 22 \\
\hline
\end{tabular}


J. Computer Sci., 8 (5): 716-720, 2012

Table 2: Analysis of the framework with respect to Experts for Ureteric and Bladder Calculi patients

\begin{tabular}{|c|c|c|c|c|c|c|c|c|}
\hline Experts & $\begin{array}{l}\text { Total number of } \\
\text { calculi detected }\end{array}$ & f Calculi & $\begin{array}{l}\text { Automatic seed } \\
\text { selected region } \\
\text { growing } \\
\text { method with } \\
\text { shadow detection }\end{array}$ & $\begin{array}{l}\text { Pyramidal seeded } \\
\text { region growing with } \\
\text { shadow detection }\end{array}$ & $\begin{array}{l}\text { Region growing } \\
\text { algorithm } \\
\text { with shadow detection }\end{array}$ & $\begin{array}{l}\text { Split and merge } \\
\text { with shadow detection }\end{array}$ & $\begin{array}{l}\text { Active contour } \\
\text { algorithm } \\
\text { with shadow detection }\end{array}$ & $\begin{array}{l}\text { Morphology } \\
\text { based watershed } \\
\text { segmentation with } \\
\text { shadow detection }\end{array}$ \\
\hline \multirow{3}{*}{$1,2,3$} & \multirow{3}{*}{22} & & D M & D M & D M & D M & D M & D M \\
\hline & & Calculi present & 166 & 166 & 166 & 157 & 166 & 166 \\
\hline & & Calculi absent & 06 & 16 & 06 & 26 & 16 & 06 \\
\hline
\end{tabular}

D-Detected, M-Missed

Table 3: Sensitivity and specificity analysis of ureteric and bladder calculi patients as per experts

\begin{tabular}{|c|c|c|c|c|c|}
\hline Name of the algorithm & $\begin{array}{l}\text { Sensitivity } \\
(\%)\end{array}$ & $\begin{array}{l}\text { Specificity } \\
(\%)\end{array}$ & $\begin{array}{l}\text { Positive } \\
\text { predictive } \\
\text { value (\%) }\end{array}$ & $\begin{array}{l}\text { Negative } \\
\text { predictive } \\
\text { value (\%) }\end{array}$ & $\begin{array}{l}\text { Efficiency } \\
(\%)\end{array}$ \\
\hline $\begin{array}{l}\text { Automatic seed selected region } \\
\text { growing method with shadow detection }\end{array}$ & 94.82 & 100.00 & 100.00 & 50.00 & 78.57 \\
\hline $\begin{array}{l}\text { Pyramidal seeded } \\
\text { Region growing with shadow detection }\end{array}$ & 72.72 & 100.00 & 94.10 & 50.00 & 78.57 \\
\hline Region growing algorithm with shadow detection & 72.72 & 85.71 & 100.00 & 50.00 & 75.86 \\
\hline Active contour algorithm with shadow detection & 72.72 & 85.71 & 94.12 & 50.00 & 75.86 \\
\hline Morphology based watershed & 72.72 & 100.00 & 100.00 & 50.00 & 78.57 \\
\hline Segmentation with shadow detection & & & & & \\
\hline Split and merge with shadow detection & 68.10 & 75.00 & 88.24 & 46.15 & 70.00 \\
\hline
\end{tabular}

Table 4: Average border metric and timing

\begin{tabular}{ll}
\hline Name of the algorithm & $\begin{array}{l}\text { Ureteric and } \\
\text { bladder calculi }\end{array}$ \\
\hline $\begin{array}{l}\text { Automatic seed selected region } \\
\text { growing method with shadow detection }\end{array}$ & 0.15 \\
Pyramidal seeded & \\
Region Growing with Shadow Detection (PSRG-SD) & 0.15 \\
Region Growing Algorithm with Shadow Detection (RG-SD) & 0.16 \\
Active Contour Algorithm with Shadow Detection & 0.16 \\
Morphology based watershed segmentation with shadow detection & 0.19 \\
Split and merge with shadow detection & 0.30 \\
\hline
\end{tabular}

Time considerations: The time complexities of the algorithms are tabulated in Table 4. This implementation is carried out in the Pentium IV processor in a MATLAB environment.

\section{DISCUSSION}

There are two categories of algorithms used. The algorithms that are coming under the category of automatic methods are proposed by the authors (Sridhar et al., 2002). Split and merge and the rest of the algorithms are semi-automated category of algorithms. In Pyramidal seeded region growing technique, the seeds grow independently and are merged. So the algorithm is able to detect the border more effectively. The execution time of the region-growing algorithm is kept low by the selection of pyramid data structure, where the region of interest is searched in the lesser resolution and mapped into the higher resolution only if the region of interest is present. The success of the morphology segmentation is based on the suitable structural mask. Since the shape of the calculi is unpredictable, the selection of a suitable mask is difficult. Similarly, in active contour technique, a small curve is introduced in the potential calculi region. In the absence of knowledge of the shape of the calculi, it is difficult to predict the selection of the suitable mask is difficult. Similarly, in active contour technique, a small curve is introduced in the potential calculi region. In the absence of knowledge of the shape of the calculi, it is difficult to predict the selection of the suitable snake.

\section{CONCULSION}

Most of the algorithms are able to detect the calculi successfully. But the major difference lies in the detection of the border of the calculi accurately. Therefore pyramidal seeded region growing algorithm performs consistently well in all cases.

Medical imaging applications require more reliability. Since the success of calculi detection and accuracy of the calculi boundary is more important than the execution time, the automatic method of segmenting the calculi is very effective. Hence for the segmentation of calculi in ultrasound images, the automatic method is recommended. The wider implication of the result are that the framework can be adapted to the other medical 
applications, where ultrasound images are used like construction of expert systems, which is useful to give accurate expertise relating to treatment (Liebovitz, 2009). It is also useful in constructing knowledge-based applications, where decision support or decisionmaking. It is also useful in applications related to telemedicine.

\section{REFERENCES}

Duncan, J.S. and N. Ayache, 2000. Medical image analysis: Progress over two decades and the challenges ahead. IEEE Trans, Patt. Anal. Med. Intell., 22: 85-106. DOI: 10.1109/34.824822

Liebovitz, D., 2009. Health care information technology: A cloud around silver lining? Arch. Internal Med., 169: 924-926. DOI: 10.1001/archinternmed.2009.79

Malvinder, S.P., 2004. Kidney stones. Br. Med. J., 328: 1420-1424. DOI: 10.1136/bmj.328.7453.1420

Marques, O., 2011. Practical Image and Video Processing using MATLAB. 1st Edn., John Wiley and Sons IEEE Computer Society Press, USA., ISBN-10: 111809347X, pp: 650.
Sridhar, S, Kumarvel N and Easwarakumar K.S., 2002. Segmentation of renal calculi in ultrasound images. Med. Inform. Internet. Med., 27: 229-236. DOI: 10.1080/1463923021000054217

Sridhar, S., 2011. Digital Image Processing. Oxford University Press, New Delhi.

Tamilselvi, P.R and P. Thangaraj, 2012. A Modified Watershed segmentation method to segment renal calculi in Ultrasound Kidney Images. IJIIT, 8: 4661.

Tomori, Z., J. Marcin and P. Vilim, 1999. Pyramidal seeded region growing algorithm and its use in image segmentation. Comput. Anal. Images Patt. Lecture Notes Comput. Sci., 1689: 835-835. DOI: 10.1007/3-540-48375-6_48

Umbaugh, S.E., 2010. Digital Imaging Processing and Analysis: Human and Computer Vision Applications with CVIPtools. 2nd Edn., CRC Press, New Jersey, ISBN: 9781439802052, pp: 955. 